\title{
Factores predictivos de reingreso tras un infarto agudo al miocardio atendido con código infarto
}

\author{
Predictive factors of re-entry after an acute myocardial infarction attended with \\ infarction code
}

\section{Guadalupe E. Gavilánez-Chávez ${ }^{1 *}$, William Matías-Calvo², Luis A. Bernal-Tejeda ${ }^{1}$, Luz R. Rodríguez-Rivera1, David A. Corona-Correa ${ }^{1}$, Luis A. Pulido ${ }^{1}$, Ana M. Sandoval-Moreno ${ }^{1}$ y Arnulfo H. Nava-Zavala ${ }^{3}$}

${ }^{1}$ Servicio de Urgencias, Hospital General Regional No. 46, Instituto Mexicano del Seguro Social (IMSS), Guadalajara, Jalisco; ${ }^{2}$ Servicio de Urgencias, Hospital General de Subzona No. 41, Instituto Mexicano del Seguro Social (IMSS), Huatulco, Oaxaca; ${ }^{3}$ Unidad de Investigación Biomédica No. 02, Instituto Mexicano del Seguro Social (IMSS), Centro Médico Nacional de Occidente, Guadalajara, Jalisco. México

\section{Introducción}

El infarto agudo al miocardio (IAM) es la necrosis miocárdica provocada por la obstrucción persistente del flujo coronario'. La rotura de la placa aterosclerosa es la causa en el $70 \%$ de los $\operatorname{casos}^{2}$ y la intervención médica para reperfundir es imperativa ${ }^{3,4}$. México logró reducir la mortalidad del $27.5 \%$ al $9.4 \%$ gracias a la estrategia "código infarto»5,6. El código infarto tiene como objetivo garantizar la reperfusión fibrinolítica en menos de 30 minutos o la intervención coronaria percutánea (ICP) primaria en menos de 90 minutos $^{7}$. Existen factores predictivos de reingreso por IAM atendido con código infarto que es necesario identificar.

El objetivo del presente estudio fue analizar los factores predictivos de reingreso tras un IAM atendido con estrategia código infarto.

\section{Método}

Estudio observacional de casos y controles a partir de los registros de pacientes atendidos con la estrategia código infarto del Hospital General Regional No. 46 en Guadalajara, México, de enero de 2018 a enero del
2020. Los pacientes que reingresaron en $\leq 30$ días constituyeron los casos y los pacientes que no reingresaron constituyeron los controles.

El análisis estadístico se realizó con el programa SPSS 24. Para la comparación de variables cualitativas se usó la prueba de $\chi^{2}$ o la prueba exacta de Fisher, y para las variables cuantitativas la prueba t de Student. Se tomó como significativo un valor de $p \leq 0.05$. Se calculó la odds ratio (OR) para cuantificar las asociaciones encontradas.

\section{Resultados}

Entre enero de 2018 y enero de 2020 se registraron 105 casos protocolizados con estrategia código infarto. De ellos, 94 fueron confirmados como IAM con elevación del segmento ST en la unidad de tercer nivel, por lo que fueron estos los que representaron nuestro universo de estudio. El promedio de edad de los casos fue de $64 \pm 11$ años, y el de los controles fue de $60 \pm 41.2$ años $(p=0.08)$. En cuanto al sexo, en los casos hubo $16(80 \%)$ hombres y $6(20 \%)$ mujeres, y en los controles $16(57 \%)$ hombres y $12(43 \%)$ mujeres $(p=0.35)$ (Tabla 1). La comorbilidad encontrada en los casos y en los controles se muestra en la tabla 1.

\section{Correspondencia:}

*Guadalupe E. Gavilánez-Chávez E-mail: dragavilanez@gmail.com
Fecha de recepción: 15-11-2021 Fecha de aceptación: 10-12-2021 DOI: 10.24875/REIE.21000153
Disponible en internet: 07-03-2022 Rev Educ Investig Emer. 2022;4(Supl 1):24-26 www.medicinadeemergencias.com bajo la licencia CC BY-NC-ND (http://creativecommons.org/licenses/by-nc-nd/4.0/). 
Tabla 1. Características generales y comorbilidad en casos y controles

\begin{tabular}{|c|c|c|c|}
\hline Variables & Casos $(n=22)$ & Controles ( $n=28$ ) & $\mathbf{p}^{*}$ \\
\hline $\begin{array}{l}\text { Sociodemográficas } \\
\text { Edad, años }\end{array}$ & $64 \pm 1.4(53-79)$ & $60 \pm 4.2(31-78)$ & 0.08 \\
\hline $\begin{array}{l}\text { Sexo } \\
\text { Hombres } \\
\text { Mujeres }\end{array}$ & $\begin{array}{c}16(80 \%) \\
6(20 \%)\end{array}$ & $\begin{array}{l}16(57 \%) \\
12(43 \%)\end{array}$ & 0.35 \\
\hline $\begin{array}{l}\text { Comorbilidad } \\
\text { Diabetes mellitus tipo } 2 \\
\text { Hipertensión arterial sistémica - } \\
\text { obesidad } \\
\text { Tabaquismo } \\
\text { Cardiopatía isquémica } \\
\text { Enfermedad renal crónica } \\
\text { Dislipidemia }\end{array}$ & $\begin{array}{c}13(65 \%) \\
9(45 \%) \\
7(35 \%) \\
6(30 \%) \\
2(10 \%) \\
1(5 \%) \\
4(20 \%)\end{array}$ & $\begin{array}{c}16(57 \%) \\
21(75 \%) \\
11(35 \%) \\
16(57 \%) \\
- \\
- \\
11(35 \%)\end{array}$ & $\begin{array}{l}0.20 \\
0.75 \\
0.36 \\
0.16 \\
0.01 \\
0.36 \\
0.30\end{array}$ \\
\hline
\end{tabular}

*Obtenido empleando las pruebas $t$ de Student, $\chi^{2}$ o exacta de Fisher, según proceda.

Tabla 2. Características del tratamiento de reperfusión recibido

\begin{tabular}{|l|c|c|c|}
\hline Variables & Casos $(\mathbf{n}=\mathbf{2 2})$ & Controles $(\mathbf{n}=\mathbf{2 8})$ & $\mathbf{p}^{*}$ \\
\hline Trombólisis & $12(60 \%)$ & $22(78 \%)$ & 0.60 \\
\hline Tiempo puerta-aguja, minutos, media (rango) & $41(15-120)$ & 0.19 \\
\hline Intervención coronaria percutánea & $22(100 \%)$ & $28(100 \%)$ & 0.03 \\
\hline Tiempo puerta-balón, horas, media (rango) & $6(1.5-8)$ & $7(1.5-23)$ & 0.05 \\
\hline Arterias afectadas & & & 0.64 \\
Una & $11(55 \%)$ & $20(78 \%)$ & 0.60 \\
Dos & $6(30 \%)$ & $2(7 \%)$ & 0.50 \\
\hline Tres & - & $1(3 \%)$ & $<0.001$ \\
\hline Causa de reingreso & & & \\
Causa no cardiaca & $14(70 \%)$ & $17(60 \%)$ & \\
\hline Insuficiencia cardiaca aguda & $9(45 \%)$ & $5(17 \%)$ & \\
Angina Inestable & $6(30 \%)$ & $15(53 \%)$ & \\
Reinfarto & $3(15 \%)$ & $4(14 \%)$ & - \\
Edema agudo pulmonar & $1(5 \%)$ & $1(3 \%)$ & \\
Arritmia no especificada & - & & \\
\hline
\end{tabular}

*Obtenido empleando las pruebas $t$ de Student, $\chi^{2}$ o exacta de Fisher, según proceda.

El tratamiento fibrinolítico se realizó en 12 (54.5\%) de los casos, con un promedio puerta-aguja de 41 minutos. La ICP se realizó en el 100\% (22 casos) en un tiempo promedio de 6 horas. En los controles, la fibrinólisis se realizó en 22 pacientes $(78.5 \%)(p=0.60)$, con un promedio de tiempo puerta-aguja de 65 minutos $(p=0.19)$. En los controles, la ICP se realizó en $28(100 \%)(p=0.03)$, con un tiempo promedio de 7 horas $(p=0.05)$. Se reportó lesión de una sola arteria en $11(55 \%)$ casos y $20(78 \%)$ controles $(p=0.64)$. Hubo dos o más arterias lesionadas en $6(30 \%)$ casos y $2(7 \%)$ controles $(p=0.60)$ (Tabla 2$)$.

Los principales diagnósticos identificados como causa de reingreso en los casos fueron insuficiencia cardiaca aguda en $9(45 \%)$, angina inestable en $6(30 \%)$, reinfarto en $3(15 \%)$, edema agudo pulmonar en 1 (5\%) y otras causas no cardiacas en 14 (70\%) (Tabla 2).

En las variables con significancia estadística, el valor de la OR se presenta en la tabla 3. Encontramos que tener una edad $>60$ años, tener una cardiopatía previa, tener un tiempo puerta-balón $>6$ horas y haber requerido ICP son los principales factores de riesgo asociados a reingreso a urgencias en 30 días o menos.

Como debilidad en nuestro estudio encontramos un sesgo de información y un registro de pacientes con código infarto incompleto. Como fortaleza, la investigación se realizó con información generada de la práctica clínica diaria, lo que ofrece una situación real de lo que ocurre en la atención de estos pacientes. Futuros estudios deberán enfocarse en identificar las causas de 
Rev Educ Investig Emer. 2022;4(Supl 1)

Tabla 3. Odds ratios de las variables encontradas como factor de riesgo de reingreso

\begin{tabular}{|l|c|c|c|c|}
\hline Variable & Casos $(\mathbf{n}=\mathbf{2 2})$ & Controles $(\mathbf{n}=\mathbf{2 8})$ & $\mathbf{p}^{*}$ & OR \\
\hline Edad & $64 \pm 1.4(53-79)$ & $60 \pm 4.2(31-78)$ & 0.08 & 0.70 \\
\hline Cardiopatía isquémica & $2(10 \%)$ & - & 0.01 & 2.08 \\
\hline Intervención coronaria percutánea & $22(100 \%)$ & $28(100 \%)$ & 0.03 & 0.78 \\
\hline Tiempo puerta-balón, horas, media (rango) & $6(1.5-8)$ & $7(1.5-23)$ & 0.05 & 0.27 \\
\hline
\end{tabular}

OR: odds ratio.

*Obtenido empleando las pruebas $t$ de Student, $\chi^{2}$ o exacta de Fisher, según proceda.

reingreso no cardiacas, que fueron las más frecuentes en este estudio.

\section{Conclusiones}

Tras atender a un paciente con IAM con elevación del segmento ST con estrategia código infarto deben tomarse en cuenta la edad, la cardiopatía isquémica previa al infarto y el tiempo de realización de la ICP para mantener al paciente con vigilancia y seguimiento de forma estrecha, con miras a disminuir los reingresos al servicio de urgencias.

\section{Financiamiento}

Los autores declaran no haber recibido financiamiento para este estudio.

\section{Conflictos de intereses}

Los autores declaran no tener conflicto de intereses.

\section{Responsabilidades éticas}

Protección de personas y animales. Los autores declaran que para esta investigación no se han realizado experimentos en seres humanos ni en animales.
Confidencialidad de los datos. Los autores declaran que en este artículo no aparecen datos de pacientes.

Derecho a la privacidad y consentimiento informado. Los autores declaran que en este artículo no aparecen datos de pacientes.

\section{Bibliografía}

1. White $\mathrm{H}$, Thygesen $\mathrm{K}$, Alpert JS, Jaffe A. Universal MI definition update for cardiovascular disease. Curr Cardiol Rep. 2014;16:492.

2. Berg DD, Wiviott SD, Braunwald E, Guo J, Im K, Kashani A, et al. Modes and timing of death in 66252 patients with non-ST-segment elevation acute coronary syndromes enrolled in $14 \mathrm{TIMI}$ trials. Eur Heart J. 2018;39:3810-20.

3. Ibanez B, James S, Agewall S, Antunes MJ, Bucciarelli-Ducci C, Bueno H et al. 2017 ESC Guidelines for the management of acute myocardial infarction in patients presenting with ST-segment elevation: The Task Force for the management of acute myocardial infarction in patients presenting with ST-segment elevation of the European Society of Cardiology (ESC). Eur Heart J. 2018;39:119-77.

4. Reimer KA, Lowe JE, Rasmussen MM, Jennings RB. The wavefron phenomenon of ischemic cell death. 1. Duration of coronary occlusion in dogs. Circulation. 1977;56:786-94.

5. Consejo Nacional de Evaluación de la Política de Desarrollo Social. México: Evaluación Estratégica de Protección Social; 2018. Disponible en: www.coneval.org.mx

6. Sánchez GB, Covarrubias HA, Rodríguez GP, Uribe EA, Ramírez-Arias E, Peralta MR, et al. Impacto de la implementación de Código Infarto en pacientes con infarto agudo de miocardio con elevación del segmento ST en el Hospital de Cardiología del Centro Médico Nacional Siglo XXI. Gac Med Mex. 2017;153(Supl 2):S13-7.

7. Borrayo-Sánchez G, Pérez-Rodríguez G, Martínez-Montañez OG, Almeida-Gutiérrez E, Ramírez-Arias E, Estrada-Gallegos J, et al. Protocolo para atención de infarto agudo de miocardio en urgencias. Rev Med Inst Mex Seguro Soc. 2017;55:233-46. 